\title{
AMINO ACID TRANSPORTERS IN DRUG DISCOVERY
}

\author{
Mariafrancesca Scalise, Lorena Pochini, Michele Galluccio and Cesare Indiveri \\ Department of DiBEST (Biologia, Ecologia, Scienze della Terra) Unit of Biochemistry and MolecularBiotechnology, \\ via Bucci 4C, University of Calabria, 87036 Arcavacata di Rende, Italy
}

Received 2014-07-29; Revised 2014-09-08; Accepted 2014-09-15

\begin{abstract}
Administered drugs interact with membrane transporters of epithelia, Blood Brain Barrier and other districts influencing their delivery and efficacy. Drugs can also be used as inhibitors of transporters involved in human pathology. Drug-transporter interactions are responsible of off-target effects contributing to toxicity. High Throughput Screening technologies increased the potential applications in therapy or in predicting side effects. These strategies will be helpful in reducing animal experimentation. The identification of transporters important for drug absorption, delivery and side effect production and the best technologies for studying interactions are the main goals in this field. Amino acid transporters are not yet considered in human therapy in spite of their involvement in several pathologies. The function of the amino acid transporters EAAT1, ASCT2, GLYT2, GLYT1, B0AT1, LAT1 and LAT2 is so far well characterized. Some structural data on these transporters have also been obtained by bioinformatics. Interactions of these proteins with several drugs have been well defined at the molecular level. Large scale and, in some cases, high throughput screening of pharmacological compounds make these transporters of particular interest and potential application in human health.
\end{abstract}

Keywords: Amino Acid Transporters, Drug Delivery, Pharmacokinetics, Screening, Liposome

\section{INTRODUCTION}

The assumption that the majority of administered drugs interact with membrane transporters located at the boundary between the intra end extracellular environment, became accepted over the years and now is well established (DeGorter et al., 2012; Giacomini et al., 2010; Han, 2011; Huang et al., 2008; Nakanishi and Tamai, 2011). Noteworthy, the brush border epithelia of intestine and kidney tubules have a very large surface in contact with the external environments from which drugs are absorbed after oral administration or reabsorbed after glomerular filtration. In these and in other districts, such as the Blood Brain Barrier (BBB), membrane transporters mediate delivery of most drugs influencing their efficacy (Agarwal et al., 2013; Giacomini et al., 2010; Mandery et al., 2012; Pardridge, 2012). Drugtransporter interactions play also key roles in therapeutic interventions in which inhibition of a specific transporter causes beneficial effects in human pathologies. This applies both to transporters overexpressed in pathologies such as cancer and to transporters expressed at normal levels (Ganapathy et al., 2009; Penmatsa et al., 2013).

Finally, drug-transporter interactions could lead to other unexpected and undesired effects caused byimpairment or stimulation of physiological activity of a specific transporter. These phenomena are collectively known as off-target interactions, i.e., binding to proteins different from the primary target of the drugs, which contributes in generating side Corresponding Author: Mariafrancesca Scalise, Lorena Pochini and Cesare Indiveri, Department of DiBEST (Biologia, Ecologia, Scienze della Terra) Unit of Biochemistry and MolecularBiotechnology, via Bucci 4C, University of Calabria, 87036 Arcavacata di Rende, Italy 
effects. These aspects promise great progress after developing of large scale and High Throughput Screening (HTS) of drugs. Therefore, knowledge of the interaction mechanisms of pharmacological compounds with membrane transporters can help in improving drugs bioavailability, in designing new pharmacological inhibitors, in predicting off-target interactions. All these applications are strategically important for reducing animal experimentation with terrific consequences both in ethics and costs. This research area has been acknowledged not only by academic scientists but also by regulatory and industrial bodies (Giacomini and Huang, 2013). The International Transporter Consortium (Chambers et al., 2011) provided important indications for establishing: (i) which transporters are important for drug absorption, delivery and excretion; (ii) the best technologies for studying interactions of transporters with drugs; (iii) the criteria for designing clinical studies for evaluating drug interactions with transporters. Several transporters are ascertained to be involved in drug delivery and reviewed in a number of papers (Ciarimboli, 2011; Koepsell et al., 2007; Rask-Andersen et al., 2013). More recently, regulatory guidance in considering drugtransporter interactions have been recommended (USFDA, 2012; EMA, 2012). Also the Society for Laboratory Automation and Screening (SLAS, https://www.slas.org), started to consider membrane transporters as interesting objects for HTS and application in drug discovery. Therefore, methodologies which help prediction of drug transporter interactions are very welcome. In fact, the field of membrane transporters, being a relatively recent research area, is continuously evolving due to the progress in manipulating and studying the hydrophobic membrane proteins. Consequently, also the knowledge on drugtransporter interactions and mechanisms is expanding. As an example, amino acid transporters are not yet considered among those recommended by the ITC, in spite of their strategic importance in human health and therapy. The purpose of the present review is, to trace an update on the current and potential involvement of the amino acids transporters in drug interaction. Specific reference to their involvement in widely diffused pathologies such as cancer is made.

\section{AMINO ACID TRANSPORTERS}

Several SLC families, grouped on the basis of sequence similarity, include amino acid transporters among their members. This classification had as a consequence the presence, in the same SLC family, of membrane transporters that exhibit different substrate specificity but a common evolutionary history.

The main characteristics of amino acid membrane transporters is the apparent redundancy and overlapping function. According to this, the same amino acids can be substrates of different transporters belonging to different SLCs. One typical example is the amino acid glutamine for which a complex network of membrane transporters exists. This phenomenon may find explanation on the importance of maintaining amino acid homeostasis in the whole body since these nutrients have pleiotropic roles and each tissue may have special needs to accomplish its function. A brief summary of the main functional characteristics of the SLCs families including amino acid transporter is reported in Table $\mathbf{1}$, together with references describing interactions with drugs. In spite of the mentioned redundancy, the localization in absorbing epithelia as well as in tissues with high metabolic rates, few of these proteins have been considered for interactions with drugs or in general with xenobiotics (Table 1). In addition, Package Inserts (PIs) of recently approved molecules consider organic cation or anion transporters but not amino acid ones (Agarwal et al., 2013). Therefore, the interaction of amino acid transporters with drugs is a relatively recent field of investigation that, in our opinion, will grow in the next years. An up to date of the most important achievements in the relationships of amino acid transporters with drug discovery and interaction will be provided. Among the findings summarized in references reported in Table 1, a focus will be given, together with the essential information, on those transporters for which interactions with drugs has been deeply analyzed by performing small or large scale screening of pharmacological compounds exploiting also the molecular mechanisms. The other amino acid transporters for which the information is still preliminary remain only listed in Table 1 to furnish a complete overview of status artis.

\section{INTERACTIONOF AMINO ACID TRANSPORTERS WITH DRUGS}

\subsection{SLC1A3: EAAT1}

EAAT1, also known as GLAST1belongs to SLC1 family. EAAT1 (SLC1A3) gene has been identified together with SLC1A1 (EAAT3) and SLC1A2 (EAAT2). Later, the other subtypes EAAT4 and EAAT5 were identified. These transporters are responsible of glutamate and aspartate uptake in neurons. In particular, EAAT1 besides astrocytes is mainly expressed in heart, skeletal muscle and placenta (Grewer et al., 2014). 
Mariafrancesca Scalise et al. / Current Research in Drug Discovery 1 (1): 1-16, 2014

Table 1. The basic characteristics of the amino acid transporters. The SLC classifications is reported; the major substrates of transporters are indicated; known interaction with drugs are reported with relative references (in italics those references dealt within the manuscript)

\begin{tabular}{|c|c|c|c|c|}
\hline Family & Amino acid Transporter & Aliases & Main substrate & Known drug interactions \\
\hline \multirow[t]{7}{*}{ SLC1 } & A1 & EAAT3 & Glu/Asp & (Jensen and Brauner-Osborne, 2004); \\
\hline & $\mathrm{A} 2$ & EAAT2 & Glu/Asp & (Jensen and Brauner-Osborne, 2004) \\
\hline & A3 & EAAT1 & Glu/Asp & (Jensen and Brauner-Osborne, 2004) \\
\hline & A4 & ASCT1 & $\mathrm{Ala} / \mathrm{Ser} / \mathrm{Cys} / \mathrm{Thr}$ & none \\
\hline & A5 & ASCT2 & $\mathrm{Ala} / \mathrm{Ser} / \mathrm{Gln} / \mathrm{Thr} / \mathrm{Asn}$ & (Albers et al., 2012; Oppedisano et al., 2012); \\
\hline & A6 & EAAT4 & Glu/Asp & (Zheng et al., 2010) \\
\hline & A7 & EAAT5 & Glu/Asp & none \\
\hline \multirow[t]{9}{*}{ SLC6 } & A5 & GlyT2 & Gly & (Vandenberg et al., 2014) \\
\hline & A7 & PROT & Pro & none \\
\hline & A9 & GlyT1 & Gly & (Parrott et al., 2013) (Sugane et al., 2013) \\
\hline & A14 & ATB0,+ & neutral and cationic amino acids & (Muller et al., 2014) (Karunakaran et al., 2011) \\
\hline & A15 & B0AT2 & large neutral amino acids & (Januchowski et al., 2014) \\
\hline & A17 & XT1 & neutral amino acids & none \\
\hline & A18 & B0AT3 & neutral amino acids & none \\
\hline & A19 & B0AT1 & neutral amino acids & (Pochini et al., 2014) \\
\hline & $\mathrm{A} 20$ & XT3 & Pro & none \\
\hline \multirow[t]{11}{*}{ SLC7 } & A1 & CAT-1 & cationic amino acids & (Grupper et al., 2013) (Speranza et al., 2011) \\
\hline & A2 & CAT-2 & cationic amino acids & none \\
\hline & $\mathrm{A} 3$ & CAT-3 & cationic amino acids & (Januchowski et al., 2014) \\
\hline & A5 & LAT1 & large neutral amino acids & (del Amo et al., 2008) \\
\hline & A6 & $y+$ LAT2 & cationic and neutral amino acids & none \\
\hline & A7 & $y+L A T 1$ & cationic and neutral amino acids & none \\
\hline & A8 & LAT2 & neutral amino acids & (del Amo et al., 2008) \\
\hline & A9 & $\mathrm{b} 0,+\mathrm{AT}$ & cationic and neutral amino acids & (Kadam et al., 2013) \\
\hline & A10 & Asc-1 & small neutral amino acids & (Brown et al., 2014) (Helboe et al., 2003) \\
\hline & A11 & $\mathrm{xCT}$ & CySS/Glu & (Dixon et al., 2014) (Albrecht et al., 2010) \\
\hline & A13 & AGT-1 & Asp/Glu & none \\
\hline SLC16 & A10 & MCT10 & Aromatic amino acids & (Gao et al., 2013) \\
\hline \multirow[t]{3}{*}{ SLC36 } & A1 & PAT1 & Ala & (Schioth et al., 2013); (Edwards et al., 2011) (Thwaites and Anderson, 2011) \\
\hline & $\mathrm{A} 2$ & PAT2 & Pro/Glu/Ala & (Schioth et al., 2013); (Edwards et al., 2011) (Thwaites and Anderson, 2011) \\
\hline & A4 & PAT4 & $\operatorname{Trp}$ & (Schioth et al., 2013); (Thwaites and Anderson, 2011) \\
\hline \multirow[t]{6}{*}{ SLC38 } & A1 & SNAT1 & Gln/Ala/Asn/CysHis/Ser & (Schioth et al., 2013); (Kwak et al., 2012) \\
\hline & A2 & SNAT2 & Ala/Asn/Cys/Gln Glu/his/Met/Pro Ser & (Schioth et al., 2013); (Kwak et al., 2012) \\
\hline & A3 & SNAT3 & Gln/His/Ala/Asn & (Rahamimoff et al., 2013) \\
\hline & A4 & SNAT4 & Ala/Asn/Cys/Glu Ser/Thr & (Schioth et al., 2013); (Januchowski et al., 2014) \\
\hline & A5 & SNAT5 & Gln/Asn/His/Ser & (Misiewicz et al., 2013) \\
\hline & A7 & SNAT7 & Gln/His/Ser/AlaAsn & none \\
\hline \multirow[t]{2}{*}{ SLC43 } & A1 & LAT3 & Branched chain amino acids & (Wang et al., 2013) \\
\hline & $\mathrm{A} 2$ & LAT4 & Branched chain amino acids & (Xie et al., 2013) \\
\hline
\end{tabular}

A complex electrogenic mechanism in which a glutamate anion is co-transported with three $\mathrm{Na}^{+}$ions and exchanged for one $\mathrm{K}^{+}$was described by electrophysiological measurements. This system allows glutamate uptake against its concentration gradient. Associated to $\mathrm{Na}^{+}$current, an anion permeability has also been shown even though its physiological role is still unclear (Wadiche et al., 1995). Further insights in the EAAT translocation pathway have been obtained upon homology model built on the crystallized orthologous glutamate transporter from P.horikoshii (Yernool et al., 2004). These transporters show a Gltph fold which is also shared by the ASCT transporters (Fig. 1). Even though this transporter does not couple $\mathrm{K}^{+}$counter transport to glutamate uptake, the location of substrate and $\mathrm{Na}^{+}$binding sites have been derived. Under a physiological point of view, EAAT1 together with the other glutamate transporters contribute to the regulation of synaptic neurotransmission, to the GABA supply into neurons and to the glutamine/glutamate cycle (Grewer et al., 2014). Given these functions, it is not surprising that alterations of glutamate transporters can be responsible of a wide range of diseases, such as alcohol dependence, Alzheimer disease, autism, depression, ALS, Huntington disease (Grewer et al., 2014) (and refs herein). In the case of EAAT1, a single point mutation (P290R) appears to be the genetic basis of a form of ataxia, hemiplegia and seizure, referred to as Episodic Ataxia 6 (EA6) (Jen et al., 2005). The involvement of EAATs in several 
pathologies made these proteins interesting targets for pharmacology and drug design. In particular, searching for substrate binding requirement allowed to find efficient inhibitors of transport. Inhibitors are classified as competitive or non competitive. Hydroxyaspartate (THA) and benzyl-hydroxyaspartate (TBOA) have been developed as competitive inhibitors indicating that hydrophobic bulk is important for high affinity substrate interaction. A large scale screening of more than 3000 drugs has been performed leading to design of coumarin analogues responsible of non- competitive inhibition. This result was particularly relevant because allowed, for the first time, the identification of a binding pocket specific for EAAT1 that did not overlap with EAAT2 and EAAT3 (Erichsen et al., 2010). Noteworthy, for those pathological conditions characterized by increased glutamaergic neurotransmission, the main goal would be discovery of positive allosteric modulator that enhance the glutamate uptake leading to neuroprotection. In this field a spider toxin has been associated to increase of glutamate transporter function (Fontana et al., 2007). In 2004 the first attempt of high throughput screening for EAATs has been reported. A fluorescent based assay has been developed using HEK293 cells stably transfected with EAAT1, 2 or 3 in which the transporter activity has been measured in presence of different effectors. The assay was based on measuring the charge movement associated to glutamate or aspartate translocation. The suitability of this tool has been validated by performing, in parallel, canonical experiments. Then, in the same cells the uptake of radiolabelled substrates in presence of the same inhibitors has been followed. This report was the first attempt of validating an High Throughput Screening for testing a huge number of pharmacological compounds. Interestingly, another member of SLC1 family, named SLC1A6 (EAAT4), has been considered for a drug screening (Zheng et al., 2010). This study, however, was designed to test twenty-one FDA approved drugs on three membrane transporters with different specificities in order to distinguish true inhibitory effects from false positive ascribed to cytotoxicity. In the mentioned work, four different drugs were found to inhibit EAAT4 stably over-expressed in HEK293; however, only one drug, i.e., fluvastatin targeted EAAT4 without exerting cytotoxic effect. This finding suggested a molecular scaffold suitable for designing good EAAT4 inhibitors.

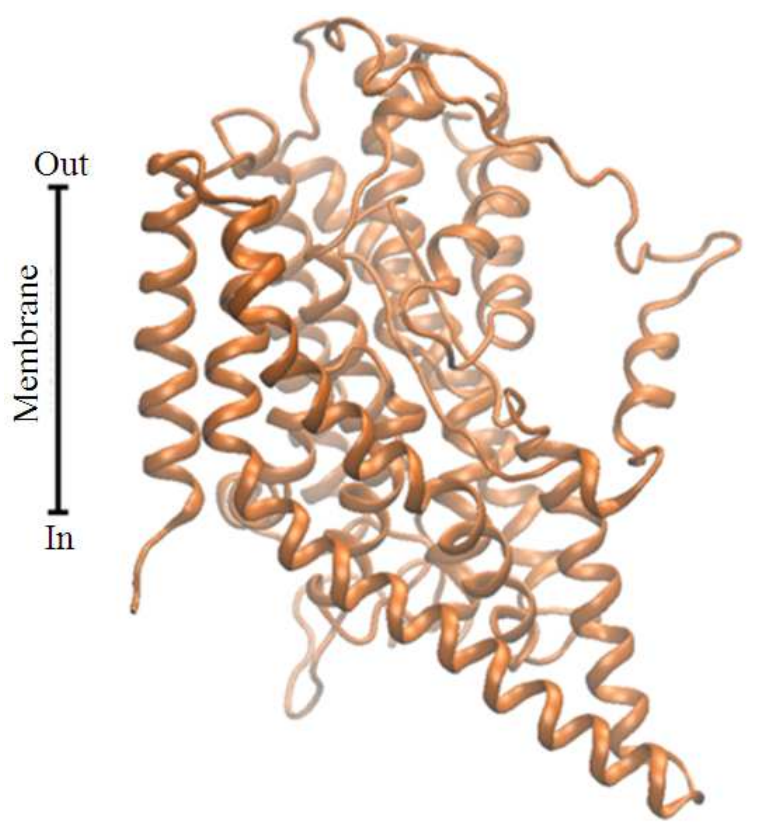

Fig. 1. Homology structural model of human ASCT2 with typical Gltph fold. The homology structural model of human ASCT2 was obtained by the Modeller 9.13 software (Sali and Blundell, 1993) using as template the structure of the glutamate transporter (Gltph) from $P$. horikoshii (PDB 1XFH). To run the software, sequences were aligned by ClustalX2 software with. pir output format. The homology model has been represented using the molecular program VMD 1.9.1. Ribbon diagram viewing from lateral side

\subsection{SLC1A5: ASCT2}

ASCT2 belongs to the SLC1 family. ASCT2 (SLC1A5) gene encodes a peptide of 541 amino acids (Kekuda et al., 1996). The expression profile of this protein is quite broad: kidney, intestine, brain, lung, skeletal muscle, placenta and pancreas (Broer and Brookes, 2001; Deitmer et al., 2003; Gliddon et al., 2009; Kekuda et al., 1996; Utsunomiya-Tate et al., 1996). ASCT2 function has been extensively studied using different experimental approaches: cell systems (Torres-Zamorano et al., 1998) as well as proteoliposome reconstituted with the rat transporter extracted from kidney brush borders (Oppedisano et al., 2004; 2007) and, more recently, with the human isoform over-expressed in P.pastoris (Pingitore et al., 2013). ASCT2 is an amino acid transporter whose acronym stands for AlaSerCysTransporter 2, even though, it is now well recognized that it has a primary role as glutamine transporter. All the experimental data coming 
from different models described similar basic properties: ASCT2 is an obligatory antiporter of neutral amino acids strictly $\mathrm{Na}^{+}$-dependent, since it does not tolerate substitution with $\mathrm{Li}^{+}$. The preferred substrates, besides glutamine, are: alanine, threonine, serine, leucine, valine, asparagine, methionine, isoleucine, tryptophan, histidine and phenylalanine, while glutamate, lysine, arginine, MeAIB and $\mathrm{BCH}$ are not substrate neither inhibitors. A functional and kinetic asymmetry has been reported: glutamine, serine, asparagine and threonine are bi-directionally transported while alanine, valine and methionine can be only inwardly transported (Pingitore et al., 2013). The overall transport reaction of hASCT2 follows a random simultaneous mechanism as recently reported in proteoliposomes (Scalise et al., 2014). A functional aspect still underneath is the electrical nature of the transport reaction; after several contradictory reports (Utsunomiya-Tate et al., 1996; Zander et al., 2013), this issue has been recently dealt with the purified hASCT2, demonstrating a positive regulation by imposed membrane potential due to an electrogenic component in the substrates translocation (Scalise et al., 2014). Under a physiological point of view, hASCT2 is involved in amino acid absorption in epithelia and other tissues contributing to the glutamine-glutamate cycle in brain and placenta. The regulation of transport activity is poorly characterized; it has been recently reported a positive regulation by intracellular $\mathrm{Na}^{+}$. Interestingly, a physical interaction with the scaffold protein PDZK1 has been described both in vitro and in vivo opening new perspectives of investigation. The regulation of ASCT2 expression is also underneath; it is linked to mTOR signaling (Nicklin et al., 2009). The interest for ASCT2 in human health arose some years ago, when a link of this transporter with cancer was discovered. Cancer cells have a special need for glutamineand hence, over-express ASCT2 and LAT1 transporters (Fuchs and Bode, 2005). Glutamine taken up with higher efficiency enters a truncated form of TCA for producing metabolic energy. This derangement occurs in cancer cells together with the well known switch towards anaerobic glucidic metabolism, known as Warburg effect (Ganapathy et al., 2009). Very recently and in line with the role of ASCT2 in cancers, it has been shown that the constitutive $\mathrm{pRb}$ degradation of cancer cells leads to enhanced expression of ASCT2 (Reynolds et al., 2014). The over-expression of ASCT2 is a hallmark of several cancers (Indiveri et al., 2014), starting investigation on inhibitors targeting specifically this protein. This kind of studies relies on the availability of three dimensional structures or homology models of the protein. As above mentioned, SLC1 family members share the Gltph fold (Yernool et al., 2004). The glutamate transporter from P.horikoshii has a trimeric quaternary structure; each protomer represents an independent transport unit, formed by eight transmembrane domains and two hairpin loops (HP1 and HP2) which are thought to be involved in substrate translocation (Boudker et al., 2007; Reyes et al., 2009). This may be valid also for ASCT2, whose rat an human (Fig. 1) homology models have been constructed using the Gltph as template (Albers et al., 2012; Oppedisano et al., 2010; Pingitore et al., 2013; Zander et al., 2013). Significant differences between the rat and humanisoforms, in a region predicted in the vicinity of the substrate binding site were found (Oppedisano et al., 2010). In addition, in the two proteins the total number of Cys residues is very different, being doubled in rat isoform. This represents an important issue since studies in proteoliposomes revealed that Cys residues are involved in substrate translocation. In one of the first study conducted on rat ASCT2 glutamine derivatives were designed as inhibitors which, however, showed very low affinity $\left(\mathrm{IC}_{50}\right.$ in the range of $500 \mu \mathrm{M}$ for the transporter (Esslinger et al., 2005). Then, a combined approach of docking and experimental validation has been used to improve this outcome; moreover, criteria for designing interactorsof this protein have been drown (Albers et al., 2012). The model predicts that small side-chain amino acids with low hydrophobicity (and their derivatives) interact with a transport competent form of the transporters behaving as substrates. While molecules with aromatic side chains interact with high affinity with the transporter behaving as inhibitor. Using the predictions, a potent inhibitor serine biphenyl-4-carboxylate was designed exhibiting an apparent $\mathrm{Ki}$ of $30 \mu \mathrm{M}$. Validation of the results were performed by measuring the anion conductance of rat ASCT2. This electrophysiological measurement however, is not the most appropriate activity assay for ASCT2, since gives indirect measurement of specific transport. According to the finding that rat ASCT2 is strongly inhibited by SH reagents (Oppedisano et al., 2010; Pingitore et al., 2013), a screening of molecules which react with $\mathrm{SH}$ functional groups was performed testing many chemical compounds chosen among known or newly synthesized molecules (Oppedisano et al., 2012). By using this approach, irreversible inhibitors, with the scaffold of dithiazoles, were identified with higher affinity, i.e., IC50 of $6 \mu \mathrm{M}$ (Oppedisano et al., 2012), respect to the substrate analogues (Albers et al., 2012). Irreversible inhibitors will have the advantage of stably inactivate the transporter with respect to competitive inhibitors, which are displaceable by the substrate. This 
property makes such molecules, potentially relevant in specifically inhibiting ASCT2 activity in cancer cells. Given the pivotal role played in cancer development/progression, the most striking interest about this transporter is towards the human protein. Notwithstanding rat transporters are considered suitable models of their human orthologs, in the case of ASCT2 this criterion is not completely fulfilled for the reasons already discussed. Therefore, the possibility of working with the recombinant human transporter is an important achievement in the research field of drug-transporter interactions (Pingitore et al., 2013). Taking into account that the proteoliposome model gives the possibility to study the specific properties of a transporter, in the absence of any interferences, large screening of drugs will be performed on hASCT2 (Scalise et al., 2013) with the aim to identify potent inhibitors potentially applicable to human therapy.

\subsection{SLC6A5 and SLC6A9: GLYT2 and GLYT1}

GlyT2 (SLC6A5) and GlyT1 (SLC6A5) belong to SLC6 family. The two genes encode transport proteins localized mainly in CNS. GlyT1 is mainly expressed in astrocytes at both pre and post synaptic terminals, but is also found in liver and pancreas (Eulenburg et al., 2005). GlyT2 has, on the contrary, exclusively a neuronal expression restricted to presynaptic terminals (Eulenburg et al., 2005). These transporters are responsible of regulating glycine concentrations in inhibitory neurons as well as in glutamatergic synapses. The reaction is energetically coupled to sodium gradient across membranes even though with two different stoichiometries: $3 \mathrm{Na}^{+} / \mathrm{Cl}^{-} / \mathrm{Gly}$ and $2 \mathrm{Na}^{+} / \mathrm{Cl}^{-} /$Gly for GlyT2 and GlyT1, respectively. This dependence on $\mathrm{Na}^{+}$gradient is responsible of the different ability of the two transporters to accumulate glycine in cells. Regulation of these transporters is tight and still under investigation: Arachidonic acid, deriving from phospholipase A2 inhibits GlyT1, $\mathrm{pH}$ modulates GlyT1 activity trough interaction with an His residue. Furthermore, $\mathrm{Zn}^{2+}$ that is co-released with glutamate inhibits specifically GlyT1 without interfering with GlyT2 activity. GlyT2 expression levels are positively modulated by membrane depolarization, $\mathrm{Ca}^{2+}$ - mediated. Some interactors of GlyTs have been described such as the PDZ protein syntenin-1 (Eulenburg et al., 2005). The interest in studying these transporters relies on their involvement in neurological diseases characterized by glycinergic neurotransmission alterations. These phenomena have been firstly described in knock-out mice for GlyTs. The animals show lethal phenotypes early after birth, with a wide range of neurological symptoms which overlap some human hereditary disease: Glycine encephalopathy and hyperkplexia, characterized by hypoglycinergic transmission. More interesting, for the wider range of applicability, is the link with schizophrenia: Inhibition of GlyT1 exerts positive effect on schizophrenic patients (Sur and Kinney, 2004).

GlyT1 and GlyT2 proteins share $50 \%$ amino acid sequence identity with $\mathrm{C}$ and $\mathrm{N}$ termini intracellularly located (Eulenburg et al., 2005) and references herein). Three-dimensional structures of these transporters is not available, even though homology models have been recently built on the basis of dopamine transporter from Drosophila melanogaster revealing a LeuT fold like B0AT1 which belongs to the same SLC family (Fig. 2). The transporters are formed by 12 transmembranes helices with ion and substrate binding sites at the bottom of a cavity. The availability of the model allowed the investigation of a novel aspect in pharmacological research, i.e., treatment of chronic pain. This is, in fact, an important issue, since the current analgesic do not work properly in mitigating pain symptoms (Vandenberg et al., 2014). Interestingly, drugs able to inhibit GlyTs can reverse the nociceptive signals. In this respect, two inhibitors specific for GlyT2 revealed to be more effective than those designed for GlyT1. These studies have been conducted on animal models showing the potential application of these inhibitors in the treatment of pain. The molecules have been docked in the tridimensional structural model of GlyTs (Vandenberg et al., 2014).

However, there are still concerns with the use of such drugs for the possible side effects; they need to be optimized in order to reduce GlyT2 activity without compromising the filling of synaptic vescicles with glycine. In the same work, the potential role of an endogenous analgesic has been evaluated. The fatty acid N-Arachidonic Glycine (NAGly) which has a structure related to the endocannabinoid anandamine, seems to alleviate pain in animal models trough interaction with GlyT2; this began the investigation on lipid inhibitors such as Oleoyl-carnitine. This area represents an open research field in drug design, even though the use of lipid-like structure may have the problem of forming micelles. This would make difficult the measurement of true drug concentration as well as the prediction of "offsite" targets (Vandenberg et al., 2014).

\subsection{SLC6A19: B0AT1}

B0AT1 belongs to the SLC6 family. B0AT1 (SLC6A19) gene encodes a transport protein localized mainly in kidney and intestine (Broer, 2006; Pramod et al., 2013; Verrey et al., 2005). B0AT1 recognizes glutamine, 
leucine, cysteine, valine, isoleucine, methionine, phenylalanine, alanine, serine and asparagine as main substrates with half saturation constants in $\mu \mathrm{M}$ range. Arginine, lysine, aspartate, glutamate and MeAIB are not substrate. Transporter function and regulation have been studied both in cell systems and in proteoliposomes. By mean of this tool the overall transport mechanism of the rat protein has been revealed as random simultaneous. In addition a kinetic asymmetry for glutamine has been reported. As expected for a transporter belonging to the $\mathrm{Na}^{+}$-dependent neurotransmitter family, the transport mechanism is strictly $\mathrm{Na}^{+}$-dependent and does not tolerate substitution with $\mathrm{Li}^{+}$. Moreover, B0AT1 mediated transport is electrogenic, not influenced by $\mathrm{Cl}^{-}$and exhibits a stoichiometry of 1aa: $1 \mathrm{Na}^{+}$(Oppedisano et al., 2011). Electrophysiology documented a channel-like activity, associated to substrate translocation, (Camargo et al., 2005). Under a physiological point of view, B0AT1 can be considered the main transporter involved in glutamine absorption and re-absorption in intestine and kidney, respectively (Bohmer et al., 2005; Broer et al., 2011; Camargo et al., 2005). B0AT1 expression is positively regulated by the Janus Kinase 2 and collectrin that increase protein abundance in the cell membrane (Bhavsar et al., 2011) and negatively regulated by leptin.
Moreover, the function of B0AT1 is up-regulated by $\mathrm{PKB} / \mathrm{Akt}$ also in tumor cells (Bogatikov et al., 2012). A specific allosteric regulation by $\mathrm{K}^{+}$has been described: $\mathrm{K}^{+}$indeed, shows a biphasic modulation at the intracellular site with stimulation up to $50 \mathrm{mM}$ internal (intracellular) $\mathrm{K}^{+}$and inhibition at higher $\mathrm{K}^{+}$ concentrations (Oppedisano and Indiveri, 2008).

The importance of B0AT1 is demonstrated by the occurrence of a mendelian disease referred to as Hartnup disease (OMIM 234500) caused by mutations of human gene encoding for this transporter.

As for the other mammalian amino acid transporters dealt within this review, three-dimensional structure of this protein is not available; however, B0AT1 displays a LeuT fold (Fig. 2). This architecture influences the substrate choice: Transported amino acid have Lconfiguration, net neutral charge and hydrophobic side chains which interact with hydrophobic pocket. Moving from the models, structure/function relationships studies have been conducted on the rat BOAT1. This protein harbors two metal binding motifs: The CXXC (containing C200/C203) and the CXXXC motifs (containing $\mathrm{C} 45 / \mathrm{C} 49$ ). These are responsible of strong inhibition by heavy metals which are harmful environmental pollutants.

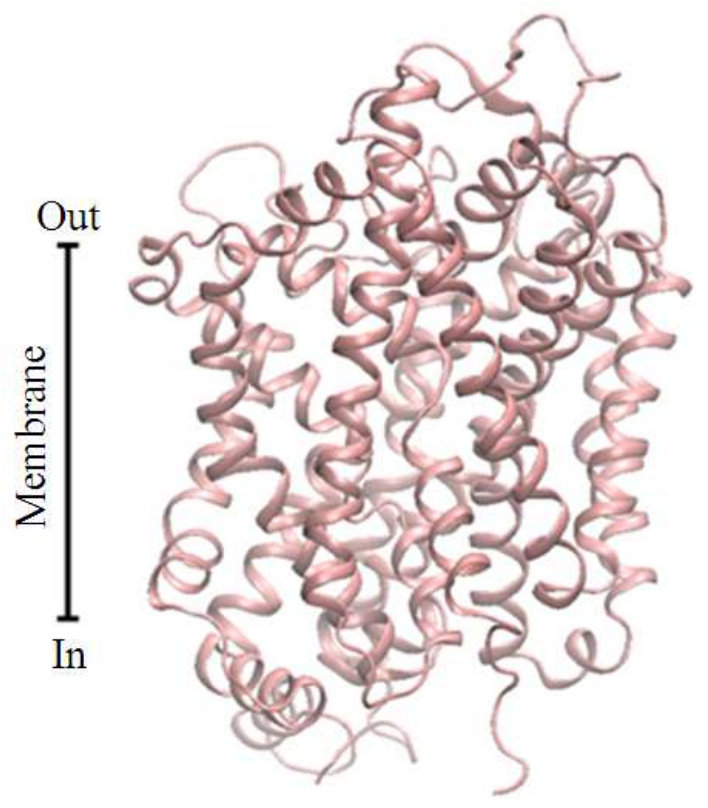

Fig. 2. Homology structural model of human B0AT1 with typical LeuT fold.The homology structural model of human B0AT1 was obtained by the Modeller 9.13 software (Sali and Blundell, 1993) using as template the structure of the Dopamine transporter from D. melanogaster (PDB 4M48). To run the software, sequences were aligned by ClustalX2 software with.pir output format. The homology model has been represented using the molecular program VMD 1.9.1. Ribbon diagram viewing from lateral side 
Regarding B0AT1 role in pharmacokinetics very little is known. Interestingly, several drugs interact with sodium-coupled transporters, with the same fold as B0AT1, such as the GABA transporter and SERT (Agarwal et al., 2013; Penmatsa et al., 2013). Taken together these observations suggested to perform a screening of different pharmaceutical compounds, commonly used in human therapy, using the B0AT1 protein from rat kidney in proteoliposomes. Among the tested compounds the anti inflammatory nimesulide is a potent inhibitor and thus, a combined proteoliposomebioinformatics experimental strategy has been adopted to reveal the molecular mechanism of inhibition (Pochini et al., 2014). The mentioned study highlighted that nimesulide exerts an inhibitory effect on glutamine uptake in a non competitive fashion since binds to an external dedicated binding site causing steric hindrance and thus, abolishing substrate translocation. Very interestingly, when the same study is conducted with respect to the other substrate of B0AT1, i.e., $\mathrm{Na}^{+}$, the inhibition followed a competitive mode.The relevance of such study is based on the high degree of identity between the rat and human isoform (more than $87 \%$ identity).

\subsection{SLC7A5 and SLC7A8: LAT1 and LAT2}

L-type Amino acid Transporters 1 (LAT1) and 2 (LAT2) belong to the large SLC7 family which includes two transporter subgroups: The Cationic Amino acid Transporters, CATs and the light subunits of Heterodimeric Amino acid Transporters, HATs. LAT1 and LAT2 are classified in this second subgroup. Their cDNAs have been isolated and identified in 1998 and in 1999 (Kanai et al., 1998; Pineda et al., 1999; Prasad et al., 1999; Segawa et al., 1999).

LAT1 and LAT2 are widely expressed (brain, ovary, testis, placenta, spleen, colon, blood-brain barrier, fetal liver, activated lymphocytes, skeletal muscle, heart, lung, thymus and kidney (Kanai et al., 1998; Yoon et al., 2005). In polarized epithelia cells, they are mainly localized at the basolateral membranes. LAT1 and LAT2 activity has been studied in cell systems. They work as heterodimers with the glycoprotein 4F2hc (CD98) encoded by SLC3A2 gene. The interaction occurs by formation of a disulfide bridge involving conserved Cys residues and it seems that the main role of $4 \mathrm{~F} 2 \mathrm{hc}$ is to route transporters to plasma membrane (Palacin and Kanai, 2004). The transport mode of LAT1 and LAT2 is quite similar, being $\mathrm{Na}^{+}$-independent antiport of amino acids, while the substrate preference is different: LAT1 mediates transport of large neutral amino acids with $\mathrm{Km}$ in micromolar range, while LAT2 mediates transport of small neutral amino acids with millimolar Km values. In cell systems, transport mediated by LAT1 and LAT2 is specifically inhibited by the non-metabolizable analogue $\mathrm{BCH}$. Under a physiological point of view, LAT1 and LAT2 are involved in equilibrating the pools of neutral amino acids, whose net uptake derive from other transporters (del Amo et al., 2008).

A role in cancer development has been clearly demonstrated for LAT1, in association to the $\mathrm{Na}^{+}$ dependent transporter ASCT2 (see paragraph "SLC1A5:ASCT2") (Fuchs and Bode, 2005). Recently, it has been reported that LAT1 promoter harbors a canonical sequence for c-myc (Hayashi et al., 2012), a known transcription factor that controls genes involved in cell cycle and metabolism. The knock out of c-myc triggers down-regulation of LAT1 expression in prostate cancer cells with subsequent decrease of proliferation (Hayashi et al., 2012). Given those information, it is not surprising that LAT1 and LAT2 are important target in pharmacology and pharmacokinetics (del Amo et al., 2008). However, the bottleneck of these studies is represented by the overlapping functions of LATs transporters and of other amino acid transporters, which hamper the measurements of affinity constants as well as the single contribution of each amino acid transporter in pharmacokinetics. Very interestingly, LAT1/CD98 and LAT2/CD98 heterodimers have been heterologously over-expressed in E.coli and P.pastoris, respectively shedding light on important functional and structural aspects (Costa et al., 2013; Galluccio et al., 2013). In particular, the availability of studying such proteins isolated from the cellular context, represents an essential condition for drug interaction analysis. Up to now the main studies conducted with LAT1 and LAT2 in cell systems have been focused on BBB, since these transporters are highly expressed in that membrane. LDOPA, used by Parkinsonian patients is transported by LAT1 and LAT2 across the BBB ( $\mathrm{Km}$ ranging from 30 to $100 \mu \mathrm{M}$ in rodents) and its absorption is decreased by high protein diet, because amino acids compete for LDOPA. Notwithstanding, there is not a definitive proof of LAT1 and LAT2 contribution in L-DOPA pharmacokinetics. The same results can be summarized for the anticancer drug mephalan and the structural analogs of GABA, gabapentin and baclofen used in epilepsy. The BBB in vitro models show transport of these drugs by LAT1 and LAT2 even though no mechanistic experiments definitively prove the role of the transporters in drug disposition and clearance. Alignment between hLAT1 and hLAT2 shows 50\% identity; 12 TMDs are predicted for the two proteins 
with intracellular N- and C-terminals. LAT1 and LAT2 show similarity with the arginine/agmantineantiporte rAdiC, the broad-specificity amino acid transporter ApcT and the glutamate/GABA antiporter from E. coli whose structure have been solved by X-ray crystallography (Ma et al., 2012; Shaffer et al., 2009). Therefore homology models have been constructed which highlight the AdiC fold for LAT transporters as shown in Fig. 3 (Geier et al., 2013). Recently, based on homology model of LAT1, some compounds have been specifically designed and tested as inhibitor of LAT1 activity in cell systems (Geier et al., 2013). This result was achieved by comparative modeling, virtual screening and experimental validation. The virtual screening has been realized using libraries of more than 12'000 compounds. The top scoring molecules have been then tested on HEK293 cells over-expressing the transporter as inhibitor of leucine uptake. Twelve hits have been characterized for their ability to cross BBB via LAT1 and to inhibit cancer cell growth. The study revealed some "scaffold" for improving drugs permeability of BBB, increasing drug disposition in CNS. Moreover, given the primary role of LAT1 in cancer development and progression, the identification of molecules with cytotoxic properties such as 3-iodo-Ltyrosine and acivicin opened new perspectives in drug design to specifically target cancer cells. Noteworthy, a recent study revealed some structural features that can discriminate LAT1 and LAT2 activities, since this is an important issue to solve in the drug design process; it has been demonstrated that contrary to LAT1, LAT2 does not accept alkyl moieties on the putative transported molecules (Khunweeraphong et al., 2012).

\section{AMINO ACID PRODRUG APPROACH}

Prodrug approach aims to increase efficacy of drugs by improving their physicochemical, biopharmaceutical or pharmacokinetic properties. Up to now $20 \%$ of all small molecule can be classified as prodrugs (Vig et al., 2013). This technique consists in facilitating drug entering into cells by means of specific transporters widely expressed in the target tissue; the targeting moiety would act as a cargo by interacting with transporter and mediating the uptake ofthe conjugated therapeutic agent across the cell membrane (Piermatti et al., 2008). This approach can be used on the one hand to enhance drug availability by increasing for example intestinal absorption (Gaucher et al., 2004; Rouquayrol et al., 2002) on the other to reduce drug affinity towards efflux transporters and thus to circumvent the phenomenon known as multi-drug resistance (Patel et al., 2013). Indeed, systemic administration of high doses of inhibitors required to inhibit $\mathrm{P}-\mathrm{gp}$ and/or MRP2 may result in relevant toxicity.

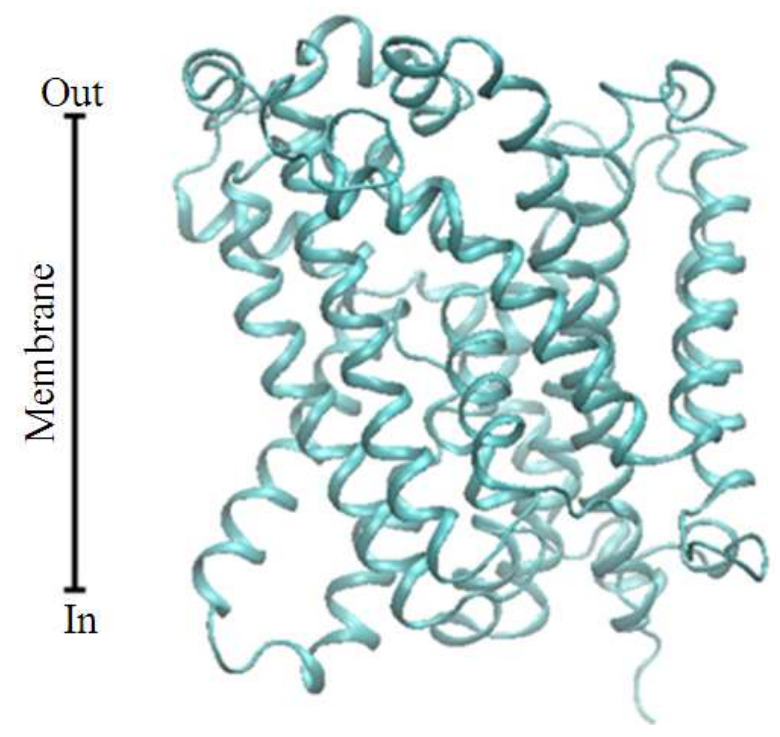

Fig. 3. Homology structural model of human LAT2 with typical AdiC fold. The homology structural model of human LAT2 was obtained by the Modeller 9.13 software (Sali and Blundell, 1993) using as template the structure of the arginine/agmantineantiporter (AdiC) from $E$. coli (PDB 3OB6). To run the software, sequences were aligned by ClustalX2 software with.pir output format. The homology model has been represented using the molecular program VMD 1.9.1. Ribbon diagram viewing from lateral side 
Moreover, this approach allows the enhancement of drug absorption in those districts where the drug delivery is impeded such as CNS, where the BBB prevents the entry of drugs. Among the molecules used as moiety, amino acids have been considered, for a number of reasons. First, the use of amino acids usually increases the water solubility of the conjugated drug through the presence of ionized carboxylate anion or ammonium cation (Vig et al., 2013). Furthermore, the use of amino acids is suitable because several membrane transporters, which play the physiological role of amino acids absorption, are expressed in intestinal epithelium. Amino acid prodrugs, thus, mimic structural features of the natural substrates. Among the amino acid transporters, intestinal mucosa expresses high levels of the protoncoupled peptide transporter 1 (PepT1, SLC15A1), of the $\mathrm{Na}^{+}$-dependent neutral amino acid transporter (ATB0,+, SLC6A14) and of LAT1 (Table 1). These transporters are characterized by high capacity, allowing rapid uptake of substrates. One of the first example of drug carried by amino acid is represented by valacyclovir and valganciclovir esterified with valine. Later on, lisdexamfetamine dimesylate has been produced as lysine amide derivative (Vig et al., 2013) and refs herein). In $\mathrm{BBB}$ the presence of amino acid transporters, like LAT1, could be exploited to allow the accumulation of a specific drug in this inaccessible district (Peura et al., 2011). Several reports have dealt with the use of natural amino acids as pro-moiety of LDOPA, 7-chlorokynurenic acid and ketoprofen via LAT1. The uptake was inhibited by 2-aminobicyclo (2, 2 , 1) heptane-2-carboxylic acid $(\mathrm{BCH})$ confirming the involvement of LAT1 ((Peura et al., 2011) and refs herein). The protease inhibitors saquinavir, indinavir and nelfinavir, largely used to treat HIV infections, have been conjugated to phenilalanine and leucine; this strategy allowed increased absorption in the intestine via LAT1 (Patel et al., 2014). Interestingly, also prodrugs with chemical structures different from amino acids have been designed to be transported by LAT1 in BBB (Peura et al., 2011). Another plasma membrane transporter used in the prodrug approach is PAT1, which belongs to SLC36 family (Table 1). This $\mathrm{H}^{+}$-coupled, $\mathrm{pH}$-dependent, low-affinity transporter is involved in alanine and small zwitterionic amino acids uptake. The relatively broad substrate specificity of PAT1 made this transporter an interesting target of administered prodrugs, like several GABA and proline related compounds which cross much easier the intestinal barrier (Thwaites and Anderson, 2011). An interesting application of amino acid prodrug strategy concerns cancer therapy and, in general, the pathological conditions in which a specific tissue over-express an amino acid transporter. Tumors, in fact, are known to need large amount of amino acids for growth and proliferation; this need is sustained by increasing expression of plasma membrane transporters as above reported (Ganapathy et al., 2009). This feature can be exploited to increase the delivery of a specific drug in the target of interest without affecting normal tissue. In breast cancer the non-nucleoside anti-cancer drug SN-38, valine-derivative, enters cells trough the transporters $\mathrm{ATB}^{0,+}$, ATA1, ATA2 and ASCT2 (Maeng et al., 2014). In prostate cancer, quinidine derivatives linked to valine and isoleucine cross cell membranes via LAT1 transporter (Patel et al., 2014). Another example is represented by increased delivery of the anti neoplastic drug floxuridine via PepT1 (Maeng et al., 2014). Even though this strategy is still at the beginning, it represents an important open field in treatment of human cancers.

\section{CONCLUSION}

As stated in the introduction, the International Transporter Consortium and FDA gave recommendations and guidelines for studying interactions of transporters with drugs (Giacomini et al., 2010). In particular, the pioneer research area of drug discovery and design is represented by identification of new targets of side effects. FDA highlighted the pivotal role played by membrane transporters in drug disposition and, then, in pharmacokinetics. Noteworthy, amino acid transporters were still not present among those listed in the mentioned studies since the information on interaction with drugs were still at the beginning. Very recent studies, described in the present review shed new light on the important contribution given by these proteins to pharmacokinetics. Notwithstanding the delay in this research field, the involvement of amino acid transporters in common and diffuse diseases, such as cancer and diabetes, makes these proteins hot spot targets for drug discovery. In this scenario, the availability of the over-expressed purified human proteins will give important efforts in discriminating specific inhibitors and/or substrates of the transporters, with important fall out in human health. 


\section{AKNOWLEDGMENT}

This study was supported by funds from: Programma Operativo Nazionale [01_00937] -MIUR "Modelli sperimentali biotecnologici integrati per lo sviluppo e la selezione di molecole di interesse per la salute dell'uomo" to CI.

\section{REFERENCES}

Agarwal, S., L. Chinn and L. Zhang, 2013. An overview of transporter information in package inserts of recently approved new molecular entities. Pharmaceutical Res., 30: 899-910. DOI: 10.1007/s11095-012-0924-0

Albers, T., W. Marsiglia, T. Thomas, A. Gameiro and C. Grewer, 2012. Defining substrate and blocker activity of Alanine-Serine-Cysteine Transporter 2 (ASCT2) ligands with novel serine analogs. Molecular Pharmacol., 81: 356-365. DOI: 10.1124/mol.111.075648

Albrecht, P., J. Lewerenz, S. Dittmer, R. Noack and P. Maher et al., 2010. Mechanisms of oxidative glutamate toxicity: The glutamate/cystine antiporter system xc-as a neuroprotective drug target. CNS Neurol. Disorders Drug Targets, 9: 373-382. DOI: 10.2174/187152710791292567

Bhavsar, S.K., Z. Hosseinzadeh, K. Merches, S. Gu and S. Broer et al., 2011. Stimulation of the amino acid transporter SLC6A19 by JAK2. Biochem. Biophys. Res. Commun., 414: 456-461. DOI: 10.1016/j.bbrc.2011.09.074

Bogatikov, E., C. Munoz, T. Pakladok, I. Alesutan and M. Shojaiefard et al., 2012. Up-regulation of amino acid transporter SLC6A19 activity and surface protein abundance by PKB/Akt and PIKfyve. Cellular physiology and biochemistry. Int. J. Exp. Cellular Physiol. Biochem. Pharmacol., 30: 15381546. DOI: $10.1159 / 000343341$

Bohmer, C., A. Broer, M. Munzinger, S. Kowalczuk and J.E. Rasko et al., 2005. Characterization of mouse amino acid transporter B0AT1 (slc6a19). Biochemical J., 389: 745-751. DOI: 10.1042/BJ20050083

Boudker, O., R.M. Ryan, D. Yernool, K. Shimamoto and E. Gouaux, 2007. Coupling substrate and ion binding to extracellular gate of a sodium-dependent aspartate transporter. Nature, 445: 387-393. DOI: 10.1038 /nature05455
Broer, A., T. Juelich, J.M. Vanslambrouck, N. Tietze and P.S. Solomon et al., 2011. Impaired nutrient signaling and body weight control in a $\mathrm{Na}+$ neutral amino acid cotransporter (Slc6a19)-deficient mouse. J. Biol. Chem., 286: 26638-26651. DOI: 10.1074/jbc.M111.241323

Broer, S. and N. Brookes, 2001. Transfer of glutamine between astrocytes and neurons. J. Neurochem., 77: 705-719. DOI: 10.1046/j.1471-4159.2001.00322.x

Broer, S., 2006. The SLC6 orphans are forming a family of amino acid transporters. Neurochem. Int., 48: 559-567. DOI: 10.1016/j.neuint.2005.11.021

Brown, J.M., L. Hunihan, M.M. Prack, D.G. Harden and J. Bronson et al., 2014. In vitro Characterization of a small molecule inhibitor of the alanine serine cysteine transporter-1 (SLC7A10). J. Neurochem., 129: 275-283. DOI: 10.1111/jnc. 12618

Camargo, S.M., V. Makrides, Virkki, L.V., I.C. Forster and F. Verrey, 2005. Steady-state kinetic characterization of the mouse B (0)AT1 sodiumdependent neutral amino acid transporter. Pflugers Archiv: Eur. J. Physiol., 451: 338-348. DOI: 10.1007/s00424-005-1455-X

Chambers, J.C., W. Zhang, J. Sehmi, X. Li and M.N. Wass et al., 2011. Genome-wide association study identifies loci influencing concentrations of liver enzymes in plasma. Nature Genet., 43: 1131-1138. DOI: 10.1038/ng.970

Ciarimboli, G., 2011. Role of organic cation transporters in drug-induced toxicity. Expert Opin. Drug Metabolism Toxicol., 7: 159-174. DOI: 10.1517/17425255.2011.547474

Costa, M., A. Rosell, E. Alvarez-Marimon, A. Zorzano and D. Fotiadis et al., 2013. Expression of human heteromeric amino acid transporters in the yeast Pichia pastoris. Protein Exp. Purif, 87: 35-40. DOI: 10.1016/j.pep.2012.10.003.

DeGorter, M.K., C.Q. Xia, J.J. Yang and R.B. Kim, 2012. Drug transporters in drug efficacy and toxicity. Annual Rev. Pharmacol. Toxicol., 52: 249-273. DOI: 10.1146/annurev-pharmtox-010611-134529

Deitmer, J.W., A. Broer and S. Broer, 2003. Glutamine efflux from astrocytes is mediated by multiple pathways. J. Neurochem., 87: 127-135. DOI: 10.1046/j.1471-4159.2003.01981.x

del Amo, E.M., A. Urtti and M. Yliperttula, 2008. Pharmacokinetic role of L-type amino acid transporters LAT1 and LAT2. Eur. J. Pharmaceutical Sci., 35: 161-174. DOI: 10.1016/j.ejps.2008.06.015 
Dixon, A.M., L. Drake, K.T. Hughes, E. Sargent and D. Hunt et al., 2014. Differential transmembrane domain GXXXG motif pairing impacts Major Histocompatibility Complex (MHC) class II structure. J. Biol. Chem., 289: 11695-11703. DOI: 10.1074/jbc.M113.516997

Edwards, N., C.M. Anderson, K.M. Gatfield, M.P. Jevons and V. Ganapathy et al., 2011. Amino acid derivatives are substrates or non-transported inhibitors of the amino acid transporter PAT2 (slc36a2). Biochimica Et Biophys. Acta, 1808: 260270. DOI: 10.1016/j.bbamem.2010.07.032.

EMA, 2012 Guideline on investigationof drug interactions

Erichsen, M.N., T.H. Huynh, B. Abrahamsen, J.F. Bastlund and C. Bundgaard et al., 2010. Structureactivity relationship study of first selective inhibitor of excitatory amino acid transporter subtype 1: 2Amino-4- (4-methoxyphenyl)-7- (naphthalen-1-yl)5-oxo-5,6,7,8-tetrahydro-4H-chrom ene-3carbonitrile (UCPH-101). J. Med. Chem., 53: 1807191. DOI: $10.1021 / \mathrm{jm} 1009154$

Esslinger, C.S., K.A. Cybulski and J.F. Rhoderick, 2005. Ngamma-aryl glutamine analogues as probes of the ASCT2 neutral amino acid transporter binding site. Bioorganic Med. Chem., 13: 1111-1118. DOI: 10.1016/j.bmc.2004.11.028

Eulenburg, V., W. Armsen, H. Betz and J. Gomeza, 2005. Glycine transporters: Essential regulators of neurotransmission. Trends Biochem. Sci., 30: 325333. doi:10.1016/j.tibs.2005.04.004

Fontana, A.C., R. De Oliveira Beleboni, M.W. Wojewodzic, W. Ferreira Dos Santos and J. Coutinho-Netto et al., 2007. Enhancing glutamate transport: Mechanism of action of Parawixin1, a neuroprotective compound from Parawixia bistriata spider venom. Molecular Pharmacol., 72: 12281237. DOI: 10.1124/mol.107.037127

Fuchs, B.C. and B.P. Bode, 2005. Amino acid transporters ASCT2 and LAT1 in cancer: Partners in crime? Seminars Cancer Biol., 15: 254-266. DOI: 10.1016/j.semcancer.2005.04.005

Galluccio, M., Pingitore, P., Scalise, M. and Indiveri, C.(2013. Cloning, large scale over-expression in E. coli and purification of the components of the human LAT 1 (SLC7A5) amino acid transporter. Protein J., 32: 442-448. DOI: 10.1007/s10930-0139503-4.
Ganapathy, V., M. Thangaraju and P.D. Prasad, 2009. Nutrient transporters in cancer: Relevance to Warburg hypothesis and beyond. Pharmacol. Therapeut., 121: 29-40. DOI: 10.1016/j.pharmthera.2008.09.005

Gao, J., H. Yang, J. Chen, J. Fang and C. Chen et al., 2013. Analysis of serum metabolites for the discovery of amino acid biomarkers and the effect of galangin on cerebral ischemia. Molecular Biosyst., 9: 2311-2321. DOI: 10.1039/c3mb70040b

Gaucher, B., M. Rouquayrol, D. Roche, J. Greiner et al., 2004. Prodrugs of HIV protease inhibitorssaquinavir, indinavir and nelfinavir-derived from diglycerides or amino acids: Synthesis, stability and anti-HIV activity. Organic Biomolecular Chem., 2: 345-357. DOI: 10.1039/b313119j

Geier, E.G., A. Schlessinger, H. Fan, J.E. Gable and J.J. Irwin et al., 2013. Structure-based ligand discovery for the large-neutral amino acid transporter 1, LAT-1. Proc. Nat. Acad. Sci. USA, 110: 5480-5485. DOI: 10.1073/Pnas. 1218165110

Giacomini, K.M. and S.M. Huang, 2013. Transporters in drug development and clinical pharmacology. Clin. Pharmacol. Therapeut., 94: 3-9. DOI: 10.1038/clpt.2013.86.

Giacomini, K.M., S.M. Huang, D.J. Tweedie, L.Z. Benet and K.L. Brouwer et al., 2010. Membrane transporters in drug development. Nat. Rev. Drug Discovery, 9: 215-236. DOI: 10.1038/nrd3028

Gliddon, C.M., Z. Shao, J.L. LeMaistre and C.M. Anderson, 2009. Cellular distribution of the neutral amino acid transporter subtype ASCT2 in mouse brain. J. Neurochem., 108: 372-383. DOI: 10.1111/j.1471-4159.2008.05767.x

Grewer, C., A. Gameiro and T. Rauen, 2014. SLC1 glutamate transporters. Pflugers Archiv: Eur. J. Physiol., 466: 3-24. DOI: 10.1007/s00424-0131397-7

Grupper, A., M. Shashar, D. Bahry, Y. Pri-Paz, O. Ben Tur et al., 2013. Cyclosporine attenuates arginine transport, in human endothelial cells, through modulation of cationic amino acid transporter-1. Am. J. Nephrol., 37: 613-619. DOI: 10.1159/000350614

Han, H.K., 2011. Role of transporters in drug interactions. Arch. Pharmacal Res., 34: 18651877. DOI: $10.1007 / \mathrm{s} 12272-011-1107-y$ 
Hayashi, K., P. Jutabha, H. Endou and N. Anzai, 2012. c-Myc is crucial for the expression of LAT1 in MIA Paca-2 human pancreatic cancer cells. Oncol. Rep., 28: 862-866. DOI: 10.3892/or.2012.1878

Helboe, L., J. Egebjerg, M. Moller and C. Thomsen, 2003. Distribution and pharmacology of alanineserine-cysteine transporter 1 (asc-1) in rodent brain. Eur. J. Neurosci., 18: 2227-2238. DOI: 10.1046/j.1460-9568.2003.02966.x

Huang, S.M., J.M. Strong, L. Zhang, K.S. Reynolds and S. Nallani et al., 2008. New era in drug interaction evaluation: US Food and Drug Administration update on CYP enzymes, transporters and the guidance process. J. Clin. Pharmacol., 48: 662-670. DOI: $10.1177 / 0091270007312153$

Indiveri, C., L. Pochini, M. Galluccio and M. Scalise, 2014. SLC1A5 (solute carrier family 1 (neutral amino acid transporter), member 5). Atlas of Genetics and Cytogenetics in Oncology and Haematology.

Januchowski, R., K. Wojtowicz, M. Andrzejewska and M. Zabel, 2014. Expression of MDR1 and MDR3 gene products in paclitaxel-, doxorubicin and vincristine-resistant cell lines. Biomed. Pharmacotherapy Biomed. Pharmacotherapie, 68: 111-117. DOI: 10.1016/j.biopha.2013.09.004

Jen, J.C., J. Wan, T.P. Palos, B.D. Howard and R.W. Baloh, 2005. Mutation in the glutamate transporter EAAT1 causes episodic ataxia, hemiplegia and seizures. Neurology, 65: 529-534. DOI: 10.1212/01.wnl.0000172638.58172.5a

Jensen, A.A. and H. Brauner-Osborne, 2004. Pharmacological characterization of human excitatory amino acid transporters EAAT1, EAAT2 and EAAT3 in a fluorescence-based membrane potential assay. Biochem. Pharmacol., 67: 21152127. DOI: 10.1016/j.bcp.2004.02.013

Kadam, R.S., S.K. Vooturi and U.B. Kompella, 2013. Immunohistochemical and functional characterization of peptide, organic cation, neutral and basic amino acid and monocarboxylate drug transporters in human ocular tissues. Drug Metabolism Disposit., 41: 466-474. DOI: 10.1124/dmd.112.045674

Kanai, Y., H. Segawa, K. Miyamoto, H. Uchino and E. Takeda et al., 1998. Expression cloning and characterization of a transporter for large neutral amino acids activated by the heavy chain of $4 \mathrm{~F} 2$ antigen (CD98). J. Biol. Chem., 273: 23629-23632.
Karunakaran, S., S. Ramachandran, V. Coothankandaswamy, S. Elangovan and E. Babu et al., 2011. SLC6A14 (ATB0,+) protein, a highly concentrative and broad specific amino acid transporter, is a novel and effective drug target for treatment of estrogen receptor-positive breast cancer. J. Biol. Chem., 286: 31830-31838. DOI: 10.1074/jbc.M111.229518

Kekuda, R., P.D. Prasad, Y.J. Fei, V. TorresZamorano and S. Sinha et al., 1996. Cloning of the sodium-dependent, broad-scope, neutral amino acid transporter Bo from a human placental choriocarcinoma cell line. J. Biol. Chem., 271: 18657-18661.

Khunweeraphong, N., S. Nagamori, P. Wiriyasermkul, Y. Nishinaka and P. Wongthai et al., 2012. Establishment of stable cell lines with high expression of heterodimers of human $4 \mathrm{~F} 2 \mathrm{hc}$ and human amino acid transporter LAT1 or LAT2 and delineation of their differential interaction with alpha-alkyl moieties. J. Pharmacol. Sci., 119: 368-380.

Koepsell, H., K. Lips and C. Volk, 2007. Polyspecific organic cation transporters: Structure, function, physiological roles and biopharmaceutical implications. Pharmaceutical Res., 24: 1227-1251. DOI: $10.1007 / \mathrm{s} 11095-007-9254-\mathrm{Z}$

Kwak, E.Y., M.K. Choi, S.G. Yang, C.K. Shim and W.S. Shim, 2012. Investigation into the Efficacy of Val-SN-38, a Valine-Ester Prodrug of the AntiCancer Agent SN-38. Biomolecules Therapeut., 20: 326-331. DOI: 10.4062/biomolther.2012.20.3.326

Ma, D., P. Lu, C. Yan, C. Fan and P. Yin et al., 2012. Structure and mechanism of a glutamate-GABA antiporter. Nature, 483: 632-636. DOI: 10.1038/nature10917

Maeng, H.J., E.S. Kim, C. Chough, M. Joung and J.W. Lim et al., 2014. Addition of amino acid moieties to lapatinib increases the anti-cancer effect via amino acid transporters. Biopharmaceut. Drug Disposit., 35: 60-69. DOI: 10.1002/bdd.1872

Mandery, K., H. Glaeser and M.F. Fromm, 2012. Interaction of innovative small molecule drugs used for cancer therapy with drug transporters. British J. Pharmacol., 165: 345-362. DOI: 10.1111/j.1476-5381.2011.01618.x

Misiewicz, M., M.A. Dery, B. Foveau, J. Jodoin and D. Ruths et al., 2013. Identification of a novel endoplasmic reticulum stress response element regulated by XBP1. J. Biol. Chem., 288: $20378-$ 20391. DOI: $10.1074 /$ jbc.M113.457242 
Muller, A., A. Chiotellis, C. Keller, S.M. Ametamey and R. Schibli et al., 2014. Imaging tumour ATB0,+ transport activity by PET with the cationic amino acid O-2 ((2-[18F]fluoroethyl)methylamino)ethyltyrosine. Molecular Imag. Biol., 16: 412-420. DOI: 10.1007/s 11307-013-0711-2

Nakanishi, T. and I. Tamai, 2011. Solute carrier transporters as targets for drug delivery and pharmacological intervention for chemotherapy. J. Pharmaceutical Sci., 100: 3731-3750. DOI: 10.1002/jps. 22576

Nicklin, P., P. Bergman, B. Zhang, E. Triantafellow and H. Wang et al., 2009. Bidirectional transport of amino acids regulates mTOR and autophagy. Cell, 136: 521-534. DOI: 10.1016/j.cell.2008.11.044

Oppedisano, F. and C. Indiveri, 2008. Reconstitution into liposomes of the $\mathrm{B}$ degrees-like glutamineneutral amino acid transporter from renal cell plasma membrane. Biochim. Biophys. Acta, 1778: 2258-2265. DOI: 10.1016/j.bbamem.2008.05.011

Oppedisano, F., L. Pochini, M. Galluccio and C. Indiveri, 2007. The glutamine/amino acid transporter (ASCT2) reconstituted in liposomes: Transport mechanism, regulation by ATP and characterization of the glutamine/glutamate antiport. Biochim. Biophys. Acta, 1768: 291-298. DOI: 10.1016/j.bbamem.2006.09.002

Oppedisano, F., L. Pochini, M. Galluccio, M. Cavarelli and C. Indiveri, 2004. Reconstitution into liposomes of the glutamine/amino acid transporter from renal cell plasma membrane: Functional characterization, kinetics and activation by nucleotides. Biochim. Biophys. Acta, 1667: 122-131. DOI: 10.1016/j.bbamem.2004.09.007

Oppedisano, F., L. Pochini, S. Broer and C. Indiveri, 2011. The B degrees AT1 amino acid transporter from rat kidney reconstituted in liposomes: Kinetics and inactivation by methylmercury. Biochim. Biophys. Acta, 1808: 2551-2558. DOI: 10.1016/j.bbamem.2011.05.011

Oppedisano, F., M. Catto, P.A. Koutentis, O. Nicolotti and L. Pochini et al., 2012. Inactivation of the glutamine/amino acid transporter ASCT2 by 1,2,3-dithiazoles: Proteoliposomes as a tool to gain insights in the molecular mechanism of action and of antitumor activity. Toxicol. Applied Pharmacol., 265: 93-102. DOI: 10.1016/j.taap.2012.09.011
Oppedisano, F., M. Galluccio and C. Indiveri, 2010. Inactivation by $\mathrm{Hg} 2+$ and methylmercury of the glutamine/amino acid transporter (ASCT2) reconstituted in liposomes: Prediction of the involvement of a CXXC motif by homology modelling. Biochem. Pharmacol., 80: 1266-1273. DOI: 10.1016/j.bcp.2010.06.032

Palacin, M. and Y. Kanai, 2004. The ancillary proteins of HATs: SLC3 family of amino acid transporters. Pflugers Archiv: Eur. J. Physiol., 447: 490-494. DOI: $10.1007 / \mathrm{s} 00424-003-1062-7$

Pardridge, W.M., 2012. Drug transport across the bloodbrain barrier. J. Cerebral Blood Flow Metabolism: Official J. Int. Society Cerebral Blood Flow Metabolism, 32: 1959-1972. DOI: 10.1038/jcbfm.2012.126

Parrott, N., D. Hainzl, D. Alberati, C. Hofmann and R. Robson et al., 2013. Physiologically based pharmacokinetic modelling to predict single- and multiple-dose human pharmacokinetics of bitopertin. Clin. Pharmacokinet., 52: 673-683. DOI: 10.1007/s40262-013-0061-X

Patel, M., N. Mandava, M. Gokulgandhi, D. Pal and A.K. Mitra, 2014. Amino acid prodrugs: An approach to improve the absorption of HIV-1 protease inhibitor, Lopinavir. Pharmaceuticals (Basel), 7: 433-452. DOI: 10.3390/ph7040433

Patel, M., P. Dalvi, M. Gokulgandhi, S. Kesh and T. Kohli et al., 2013. Functional characterization and molecular expression of Large neutral Amino Acid Transporter (LAT1) in human prostate cancer cells. Int. J. Pharmaceut, 443: 245-253. DOI: 10.1016/j.ijpharm.2012.12.029

Penmatsa, A., K.H. Wang and E. Gouaux, 2013. X-ray structure of dopamine transporter elucidates antidepressant mechanism. Nature, 503: 85-90. DOI: 10.1038/nature 12533

Peura, L., K. Malmioja, K. Laine, J. Leppanen and M. Gynther et al., 2011. Large Amino acid Transporter 1 (LAT1) prodrugs of valproic acid: New prodrug design ideas for central nervous system delivery. Molecular Pharmaceut., 8: 1857-1866. DOI: 10.1021/mp2001878

Piermatti, O., F. Fringuelli, L. Pochini, C. Indiveri and C.A. Palmerini, 2008. Synthesis and characterization of carnitine nitro-derivatives. Bioorganic Medicinal Chem., 16: 1444-1451. DOI: 10.1016/j.bmc.2007.10.053 
Pineda, M., E. Fernandez, D. Torrents, R. Estevez and C. Lopez et al., 1999. Identification of a membrane protein, LAT-2, that Co-expresses with 4F2 heavy chain, an L-type amino acid transport activity with broad specificity for small and large zwitterionic amino acids. J. Biol. Chem., 274: 19738-19744. DOI: 10.1074/jbc.274.28.19738

Pingitore, P., L. Pochini, M. Scalise, M. Galluccio and K. Hedfalk et al., 2013. Large scale production of the active human ASCT2 (SLC1A5) transporter in Pichia pastoris--functional and kinetic asymmetry revealed in proteoliposomes. Biochim. Biophys. Acta, 1828: 2238-2246. DOI: 10.1016/j.bbamem.2013.05.034

Pochini, L., A. Seidita, C. Sensi, M. Scalise, I. Eberini and C. Indiveri, 2014. Nimesulide binding site in the B0AT1 (SLC6A19) amino acid transporter. Mechanism of inhibition revealed by proteoliposome transport assay and molecular modelling. Biochemical Pharmacol., 89: 422-430. DOI: 10.1016/j.bcp.2014.03.014

Pramod, A.B., J. Foster, L. Carvelli and L.K. Henry, 2013. SLC6 transporters: Structure, function, regulation, disease association and therapeutics. Molecular Aspects Med., 34: 197-219. DOI: 10.1016/j.mam.2012.07.002

Prasad, P.D., H. Wang, W. Huang, R. Kekuda and D.P. Rajan et al., 1999. Human LAT1, a subunit of system L amino acid transporter: Molecular cloning and transport function. Biochemical Biophys. Res. Commun., 255: 283-288. DOI: 10.1006/bbrc. 1999.0206

Rahamimoff, H., B. Elbaz, M. Valitsky, M. Khatib and M. Eskin-Schwartz et al., 2013. Immunosuppressive drugs, immunophilins and functional expression of NCX isoforms. Adv. Exp. Medicine Biol., 961: 275-287. DOI: 10.1007/978-1-4614-4756-6_23

Rask-Andersen, M., S. Masuram, R. Fredriksson and H.B. Schioth, 2013. Solute carriers as drug targets: Current use, clinical trials and prospective. Molecular Aspects Med., 34: 702710. DOI: 10.1016/j.mam.2012.07.015

Reyes, N., C. Ginter and O. Boudker, 2009. Transport mechanism of a bacterial homologue of glutamate transporters. Nature, 462: 880-885. DOI: 10.1038/nature08616

Reynolds, M.R., A.N. Lane, B. Robertson, S. Kemp and Y. Liu et al., 2014. Control of glutamine metabolism by the tumor suppressor $\mathrm{Rb}$. Oncogene, 33: 556-566. DOI: 10.1038/onc. 2012.635
Rouquayrol, M., B. Gaucher, D. Roche, J. Greiner and P. Vierling, 2002. Transepithelial transport of prodrugs of the HIV protease inhibitors saquinavir, indinavir and nelfinavir across Caco-2 cell monolayers. Pharmaceutical Res., 19: 1704-1712. DOI: 10.1023/A:1020913631309

Sali, A. and T.L. Blundell, 1993. Comparative protein modelling by satisfaction of spatial restraints. J. Mol. Biol., 234: 779-815. PMID: 8254673

Scalise, M., L. Pochini, N. Giangregorio, A. Tonazzi and C. Indiveri, 2013. Proteoliposomes as tool for assaying membrane transporter functions and interactions with xenobiotics. Pharmaceutics, 5: 472-497. DOI: 10.3390/pharmaceutics5030472

Scalise, M., L. Pochini, S. Panni, P. Pingitore and K. Hedfalk et al., 2014. Transport mechanism and regulatory properties of the human amino acid transporter ASCT2 (SLC1 A5). Amino Acids. DOI: 10.1007/s00726-014-1808-x.

Schioth, H.B., S. Roshanbin, M.G. Hagglund and R. Fredriksson, 2013. Evolutionary origin of amino acid transporter families SLC32, SLC36 and SLC38 and physiological, pathological and therapeutic aspects. Molecular Aspects Med., 34: 571-585. DOI: 10.1016/j.mam.2012.07.012

Segawa, H., Y. Fukasawa, K. Miyamoto, E. Takeda and H. Endou et al., 1999. Identification and functional characterization of a $\mathrm{Na}+$-independent neutral amino acid transporter with broad substrate selectivity. J. Biol. Chem., 274: 19745-19751. DOI: 10.1074/jbc.274.28.19745

Shaffer, P.L., A. Goehring, A. Shankaranarayanan and E. Gouaux, 2009. Structure and mechanism of a Na+independent amino acid transporter. Science, 325: 1010-1014. DOI: 10.1126/science. 1176088

Speranza, L., S. Franceschelli, N. D'Orazio, R. Gaeta and T. Bucciarelli et al., 2011. The biological effect of pharmacological treatment on dimethylaminohydrolases (DDAH-1) and cationic amino acid transporter-1 (CAT-1) expression in patients with acute congestive heart failure. Microvascular Res., 82: 391-396. DOI: 10.1016/j.mvr.2011.06.003

Sugane, T., T. Tobe, W. Hamaguchi, I. Shimada and K. Maeno et al., 2013. Atropisomeric 4-phenyl-4H1,2,4-triazoles as selective glycine transporter 1 inhibitors. J. Med. Chem., 56: 5744-5756. DOI: 10.1021/jm400383w 
Sur, C. and G.G. Kinney, 2004. The therapeutic potential of glycine transporter-1 inhibitors. Expert Opin. Investigat. Drugs, 13: 515-521. DOI: 10.1517/13543784.13.5.515

Thwaites, D.T. and C.M. Anderson, 2011. The SLC36 family of proton-coupled amino acid transporters and their potential role in drug transport. Brit. J. Pharmacol., 164: 1802-1816. DOI: 10.1111/j.1476-5381.2011.01438.x

Torres-Zamorano, V., F.H. Leibach and V. Ganapathy, 1998. Sodium-dependent homo- and hetero-exchange of neutral amino acids mediated by the amino acid transporter ATB degree. Biochemical Biophys. Res. Commun., 245: 824829. DOI: $10.1006 /$ bbrc.1998.8434

USFDA, 2012. Guidance forindustry-drug interaction studies, study design, data analysis, implications for dosing and labeling recommendations. US Food and Drug Administration.

Utsunomiya-Tate, N., H. Endou and Y. Kanai, 1996. Cloning and functional characterization of a system ASC-like Na+-dependent neutral amino acid transporter. J. Biol. Chem., 271: 1488314890. DOI: $10.1074 /$ jbc.271.25.14883

Vandenberg, R.J., R.M. Ryan, J.E. Carland, W.L. Imlach and M.J. Christie, 2014. Glycine transport inhibitors for the treatment of pain. Trends Pharmacol. Sci. DOI: 10.1016/j.tips.2014.05.006

Verrey, F., Z. Ristic, E. Romeo, T. Ramadan and V. Makrides et al., 2005. Novel renal amino acid transporters. Ann. Rev. Physiol., 67: 557-572. DOI: 10.1146/annurev.physiol.67.031103.153949

Vig, B.S., K.M. Huttunen, K. Laine and J. Rautio, 2013. Amino acids as promoieties in prodrug design and development. Adv. Drug Delivery Rev., 65: 1370-1385. DOI: 10.1016/j.addr.2012.10.001
Wadiche, J.I., S.G. Amara and M.P. Kavanaugh, 1995. Ion fluxes associated with excitatory amino acid transport. Neuron, 15: 721-728. DOI: 10.1016/0896-6273(95)90159-0

Wang, Q., J. Tiffen, C.G. Bailey, M.L. Lehman and W. Ritchie et al., 2013. Targeting amino acid transport in metastatic castration-resistant prostate cancer: Effects on cell cycle, cell growth and tumor development. J. Nat. Cancer Inst., 105: 1463-1473. DOI: 10.1093/jnci/djt241

Xie, X.L., A. Kakehashi, M. Wei, S. Yamano and M. Takeshita et al., 2013. L-Leucine and Lisoleucine enhance growth of $\mathrm{BBN}$-induced urothelial tumors in the rat bladder by modulating expression of amino acid transporters and tumorigenesis-associated genes. Food Chemical Toxicol., 59: 137-144. DOI: 10.1016/j.fct.2013.05.044

Yernool, D., O. Boudker, Y. Jin and E. Gouaux, 2004. Structure of a glutamate transporter homologue from Pyrococcus horikoshii. Nature, 431: 811818. DOI: $10.1038 /$ nature 03018

Yoon, J.H., I.J. Kim, H. Kim, H.J. Kim and M.J. Jeong et al., 2005. Amino acid transport system L is differently expressed in human normal oral keratinocytes and human oral cancer cells. Cancer Lett., 222: 237-245. DOI: 10.1016/j.canlet.2004.09.040

Zander, C.B., T. Albers and C. Grewer, 2013. Voltage-dependent processes in the electroneutral amino acid exchanger ASCT2. J. General Physiol., 141: 659-672. DOI: 10.1085/jgp.201210948

Zheng, X., L. Diao, S. Ekins and J.E. Polli, 2010. Why we should be vigilant: Drug cytotoxicity observed with in vitro transporter inhibition studies. Biochemical Pharmacol., 80: 1087-1092. DOI: $10.1016 /$ j.bcp.2010.06.012 